\title{
Outage Probability Analysis of Dual-hop Full-Duplex Decode-and-Forward Relaying over Generalized Multipath Fading Conditions
}

\author{
Mulugeta K. Fikadu ${ }^{1}$, Paschalis C. Sofotasios ${ }^{1,2}$, Mikko Valkama ${ }^{1}$, Sami Muhaidat ${ }^{3,4}$, Qimei Cui ${ }^{5}$ \\ and George K. Karagiannidis ${ }^{2,3}$
}

\footnotetext{
${ }^{1}$ Department of Electronics and Communications Engineering, Tampere University of Technology, 33101 Tampere, Finland. e-mail: \{mulugeta.fikadu; paschalis.sofotasios; mikko.e.valkama\} @tut.fi

${ }^{2}$ Department of Electrical and Computer Engineering, Aristotle University of Thessaloniki, 54124 Thessaloniki, Greece. e-mail: \{sofotasios; geokarag\} @auth.gr

${ }^{3}$ Department of Electrical and Computer Engineering, Khalifa University, PO Box 127788, Abu Dhabi, UAE.

${ }^{4}$ Department of Electronic Engineering, University of Surrey, GU2 7XH, Guildford, UK. e-mail: muhaidat@ieee.org

${ }^{5}$ Wireless Technology Innovation Institute, Beijing University of Posts and Telecommunications, 100876 Beijing, China. e-mail: cuiqimei@bupt.edu.cn
}

\begin{abstract}
The present paper analyzes the outage probability of full-duplex (FD) regenerative relay systems over multipath fading channels. Unlike the majority of investigations that assume basic symmetric fading conditions, this analysis considers asymmetric generalized fading conditions, which are more realistic as they are encountered more often in practical wireless transmissions. To this end, it is assumed that the source-relay and sourcedestination links are subject to $\kappa-\mu$ multipath fading conditions, which can represent generalized line-of-sight communication scenarios; on the contrary, the relay-to-destination link is subject to $\eta-\mu$ fading conditions that typically represent generalized nonline-of-sight communication scenarios. Novel analytic expressions are derived for the outage probability (OP) of the considered FD relaying system. These expressions are given in closed-form and have a relatively tractable algebraic form which renders them convenient to handle both analytically and numerically. To this effect, they are subsequently employed in analyzing the corresponding performance for various communication scenarios. It is shown that the OP of the FD relay system is, as expected, highly dependent upon the severity of fading, the relay self-interference and the interference from the direct link. Furthermore, it is shown that at relatively high average signal-tonoise ratio values, the outage probability at low fading severity and at high relay self interference outperforms the respective performance for the case of high fading severity, but with low relay self-interference. Based on this, the offered results can be useful in the design and deployment of future full-duplex based cooperative communication systems.
\end{abstract}

\section{INTRODUCTION}

Cooperative communications have experienced significant advancements over the past decade and constitute considerable candidates for the next generation of wireless communication systems [1]-[7]. This is largely based on their distinct ability to extend network coverage, improve transmission reliability, enhance channel capacity and reduce power consumption, e.g. see [8]-[27] and the references therein. As a result, such technologies have been adopted in recent communication standards such as, IEEE 802.16j WiMAX and 3GPP Long Term Evolution (LTE) - Advanced standard [28]. It is recalled that conventional relay communication systems are typically based on half-duplex (HD) transmission which practically constitutes a non-negligible constraint as the involved relay nodes receive and transmit signals on orthogonal channels [29]-[32], which results to inefficient utilization of the system resources that ultimately incur considerable performance losses.

Nevertheless, it was recently shown that the above issue can be effectively overcome by means of full-duplex (FD) relaying, which has been proposed as an effective alternative architecture that allows relay(s) to receive and transmit at the same frequency band and time. Yet, this method is exploited at a cost of relay self-interference (RSI) that leaks between the transmit and receiver input. However, recent studies have extensively shown that FD relaying strategies are still feasible and can perform effectively even in the presence of high selfinterference levels, see e.g. [33]-[38] and the reference therein. Motivated by this, Baranwal et al. [39] derived the outage probability (OP) of a multihop full-duplex relaying (FDR) system by considering the RSI and interference from adjacent terminals over Rayleigh fading channels. In the same context, Day et al. [40] derived tight upper and lower bounds on the end-to-end achievable rate of decode-and-forward (DF) based FD multiple-input multiple-output (MIMO) relay systems. Likewise, Altieri et al. [41] analyzed the outage probability performance of FD interference-limited relaying for DF and compress-and-forward $(\mathrm{CF})$ schemes for the case of conven- 
tional multipath fading and path loss effects.

It is also recalled that fading phenomena affects significantly the performance of both conventional and emerging communication systems, which has led to the proposition of several fading models [42]-[53]. However, considering advanced fading models is typically cumbersome due to the presence of complex integrals that involve elementary and/or special functions [54]-[64] and the references therein. Based on this, all reported analyses and investigations on FD relaying assume signal transmission over symmetric multipath fading channels, i.e., that the source-relay and relay-destination links undergo the same fading conditions. Nevertheless, this is encountered rarely in realistic communication scenarios as wireless radio propagation links are typically subject to asymmetric fading conditions. Indicative examples of such communication scenarios can be found in cases that one path corresponds to line of sight (LOS) communications in the presence of a dominant component, while another path might experience severe nonline of sight NLOS conditions. Based on this, Suraweera et al. [65] investigated the end-to-end performance of a dualhop fixed gain relaying system when the source-relay and the relay-destination channels experience Rayleigh/Rician and Rician/Rayleigh fading conditions respectively. In the same context, Jayasinghe et al. [66] analyzed the performance of dual-hop transmissions for optimal beamforming in fixed gain amplify-and-forward (AF) MIMO relaying over asymmetric fading conditions. Likewise, Peppas et al. [67] investigated the end-to-end performance over generalized small scale NLOS and LOS asymmetric fading channels.

It is noted here that the reported investigations over asymmetric fading conditions are analyzed solely in the context of HD relaying systems, which, as already mentioned, constitutes a suboptimal communication principle with non-negligible performance degradation. Motivated by this, the present work analyzes the OP in FD relay systems over generalized LOS and NLOS asymmetric fading conditions. To this end, novel analytic expressions are derived for the case that the source-relay and source-destination paths are subject to $\kappa-\mu$ fading conditions while the relay-destination link is subject to $\eta-\mu$ fading conditions. The offered analytic expressions are represented in closed-form and their validity is justified through comparisons with respective results from computer simulations. The derived expressions are subsequently employed in analyzing the OP for different communication scenarios and as expected, it is shown that the performance of the considered FD relaying is highly dependent upon the severity of the fading conditions and the levels of the involved self-interference at the relay.

The reminder of this paper is organized as follows: Section II presents the considered system and channel models, whereas Section III is devoted to the derivation of novel analytic expressions of the OP over asymmetric generalized multipath fading conditions. The corresponding numerical results are presented in Section IV while closing remarks are provided in Section V.

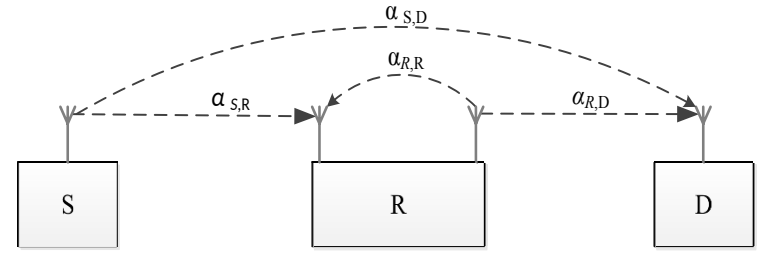

Fig. 1. Two-hop full-duplex relay system.

\section{System And Channel Model}

\section{A. System model of Full-Duplex Relay}

We consider a three-node relay network consisting of one source, $S$, a single relay, $R$, and a destination, $D$. The source and destination nodes are equipped with a single antenna while the relay has two antennas, one for receiving and one for transmitting, that implement FD mode operation as illustrated in Fig. 1. It is assumed that the relay uses DF relaying protocol to re-transmit the received signal to the destination and that reception and re-transmission occur simultaneously at the same frequency band, which ultimately induces a certain amount of relay self-interference. Based on this, the received signals at the relay and destination nodes can be expressed as

$$
y_{R}=\sqrt{P_{S}} \alpha_{S, R} x_{S}+\sqrt{P_{R}} \alpha_{R, R} x_{R}+n_{R}
$$

and

$$
y_{D}=\sqrt{P_{R}} \alpha_{R, D} x_{R}+\sqrt{P_{S}} \alpha_{S, D} x_{S}+n_{D}
$$

respectively, where $P_{S}$ and $P_{R}$ are the transmission powers at the source and relay nodes, respectively, $x_{S}$ and $x_{R}$ denote the transmitted signals from the source and relay nodes with normalized unit energy, whereas $\alpha_{S, R}, \alpha_{R, D}, \alpha_{S, D}$ and $\alpha_{R, R}$ are the fading coefficients of the $\mathrm{S} \rightarrow \mathrm{R}, \mathrm{R} \rightarrow \mathrm{D}, \mathrm{S} \rightarrow \mathrm{D}$ and $\mathrm{R} \rightarrow \mathrm{R}$ links, respectively. Also, $n_{R}$ and $n_{D}$ denote the additive white Gaussian noise (AWGN) with zero mean and variance $N_{0}$ at the relay and destination nodes, respectively, while each path is assumed to experience narrow band multipath fading conditions.

\section{B. Asymmetric Channel Model}

In the considered asymmetric fading channel model, we assume that the $S \rightarrow R$ and $S \rightarrow D$ paths are subject to $\kappa-\mu$ fading conditions, whereas the $R \rightarrow D$ path experiences $\eta-\mu$ fading conditions. It is recalled that these fading models have been shown to characterize accurately multipath fading effects in LOS and NLOS communication scenarios, respectively [68]. In addition, the relay self-interference channel is assumed to be Rayleigh distributed, which corresponds to relatively severe multipath fading conditions.

1) The $\eta-\mu$ fading model: As already mentioned, the $\eta-\mu$ distribution is a generalized fading model that provides remarkable characterization of generalized multipath fading conditions in NLOS communications. This model consists of two different formats and its signal-to-noise ratio (SNR) probability density function (PDF) is expressed as [68] 


$$
f_{\gamma}(\gamma)=\frac{2 \sqrt{\pi} \mu^{\mu+\frac{1}{2}} h^{\mu} \gamma^{\mu-\frac{1}{2}} I_{\mu-\frac{1}{2}}\left(\frac{2 \mu H \gamma}{\bar{\gamma}}\right)}{\Gamma(\mu) H^{\mu-\frac{1}{2}} \bar{\gamma}^{\mu+\frac{1}{2}} \exp \left(\frac{2 \mu \gamma h}{\bar{\gamma}}\right)}
$$

where $\gamma=|\alpha|^{2} P / N_{0}$ denotes the instantaneous SNR, $\bar{\gamma}=$ $\mathrm{E}[\gamma]$ is the average SNR per symbol, whereas $\Gamma(\cdot)$ and $I_{\nu}(\cdot)$ denote the gamma function and the modified Bessel function of the first kind, respectively [69]. The parameters $h$ and $H$ are functions of $\eta$, which varies according to the referring format. More specifically, in Format $-1, h=\left(2+\eta^{-1}+\eta\right) / 4$ and $H=$ $\left(\eta^{-1}-\eta\right) / 4$ where $0<\eta<\infty$ is the scattered-waves power ratio between the in-phase and quadrature components of each multipath cluster. On the contrary, in Format $-2, h=$ $1 /\left(1-\eta^{2}\right)$ and $H=\eta /\left(1-\eta^{2}\right)$ where $-1<\eta<1$ represents the correlation coefficient between the in-phase and quadrature components of the scattered waves in each multipath cluster. In both formats, the parameter $\mu>0$ denotes the number of mutipath clusters. Furthermore, it is recalled that the $\eta-\mu$ fading model includes as special cases the well-known Nakagami- $m$, Nakagami- $q$ (Hoyt) and Rayleigh fading distributions [68].

2) The $\kappa-\mu$ fading model: The $\kappa-\mu$ fading distribution is also a distinct fading model that differs from $\eta-\mu$ in that it accounts for generalized multipath fading conditions in LOS communications. The SNR PDF of the $\kappa-\mu$ distribution is expressed as [68]

$$
f_{\gamma}(\gamma)=\frac{\mu(\kappa+1)^{\frac{(\mu+1)}{2}} \gamma^{\frac{(\mu-1)}{2}} I_{\mu-1}\left(2 \mu \sqrt{\frac{\kappa(\kappa+1) \gamma}{\bar{\gamma}}}\right)}{\kappa^{\frac{(\mu-1)}{2}} \bar{\gamma} \frac{(\mu+1)}{2} \exp \left(\mu \kappa+\frac{\mu(\kappa+1) \gamma}{\bar{\gamma}}\right)}
$$

where $\kappa>0$ denotes the ratio between the total power of the dominant components to that of the scattered waves whereas $\mu>0$ denotes the number of multipath clusters. The $\kappa-\mu$ fading model includes the Nakagami $-m$, the Nakagami $-n$ (Rice) and Rayleigh fading models as special cases while its corresponding CDF is computed with the aid of [68, eq. (3)].

\section{Outage Probability Analysis}

In the considered DF-FD relay system, the relay is subject to self-interference while the destination is subject to interference from the signal transmission along the S-D path. To this effect, the instantaneous signal-to-interference-and-noiseratios (SINRs) at the relay and destination nodes are expressed as follows [70]

$$
\Gamma_{R}=\frac{\left|h_{S, R}\right|^{2} P_{S}}{\left|h_{R, R}\right|^{2} P_{R}+N_{0}}
$$

and

$$
\Gamma_{D}=\frac{\left|h_{R, D}\right|^{2} P_{R}}{\left|h_{S, D}\right|^{2} P_{S}+N_{0}}
$$

respectively. It is also noted that the overall outage probability in terms of the instantaneous SINR is expressed as

$$
P_{\text {out }}=\operatorname{Pr}\left(\Gamma_{R}<\Gamma_{T}\right)+\left(1-\operatorname{Pr}\left(\Gamma_{R}<\Gamma_{T}\right)\right) \operatorname{Pr}\left(\Gamma_{D}<\Gamma_{T}\right)
$$

where $\Gamma_{T}=2^{R}-1$ is the required SINR with spectral efficiency $R$ in bits/sec/Hz.

In order to derive an analytic expression for the OP of the considered system, we firstly represent (5) and (6) in terms of the equivalent SNRs, namely

$$
\Gamma_{R}=\frac{\gamma_{S, R}}{\gamma_{R, R}+1}
$$

and

$$
\Gamma_{D}=\frac{\gamma_{R, D}}{\gamma_{S, D}+1}
$$

respectively, where $\gamma_{R, R}$ is exponentially distributed with mean value $\bar{\gamma}_{R, R}$. Furthermore, $\Gamma_{R}$ and $\Gamma_{D}$ can be equivalently expressed in terms of the common RVs $X$ and $Y$ according to $Z=X /(Y+1)$. To this effect and based on the foundations of probability theory, the CDF of $Z$ can be represented as follows [71]

$$
F_{Z}(z)=\int_{0}^{\infty} F_{X}(z(y+1)) f_{Y}(y) \mathrm{d} y .
$$

Therefore, for the case of the $S \rightarrow R$ we substitute in (10) the CDF of the $\kappa-\mu$ distribution along with the PDF of Rayleigh distribution for $\gamma_{R, R}$. To this end and with the aid of [72, eq. (13)] along with long but basic algebraic manipulations, the $\mathrm{CDF}$ of $\Gamma_{R}$ can be expressed as follows

$$
\begin{aligned}
F_{Z}(z)=1 & -Q_{\mu_{S, R}}(\sqrt{b}, \sqrt{a}) \\
+ & \left(\frac{a \bar{\gamma}_{S, R}}{a \bar{\gamma}_{S, R}+2}\right)^{\mu_{S, R}} e^{\frac{1}{\bar{\gamma}_{R, R}}-\frac{b}{2+a \bar{\gamma}_{R, R}}} \\
& \times Q_{\mu_{S, R}}\left(\sqrt{\frac{a b}{a+\frac{2}{\bar{\gamma}_{R, R}}}}, \sqrt{\left.\frac{2}{\bar{\gamma}_{R, R}}+a\right)}\right.
\end{aligned}
$$

where

$$
a=\frac{2 \mu_{S, R}(1+\kappa) z}{\bar{\gamma}_{S, R}}
$$

and $b=2 \kappa \mu_{S, R}$.

In order to determine the CDF of $\Gamma_{D}$, it is firstly necessary to express the CDF of the $\eta-\mu$ distribution in terms of a simpler representation with the aid of $F_{\gamma}(z) \triangleq \int_{0}^{z} f_{\gamma}(x) \mathrm{dx}$. To this effect, by expressing the $I_{n}(\cdot)$ term in (3) according to [69, eq. (8.467], using [69, eq. (8.350.1)] along with [69, eq. (8.352. 6)] and carrying out long but basic algebraic manipulations, the SNR CDF of $\eta-\mu$ distribution for integer values of $\mu$ can be expressed as follows: 


$$
\begin{aligned}
F(z)= & \sum_{l=0}^{\mu-1} \frac{(-1)^{l}(h-H)^{l-\mu}+(-1)^{\mu}(h+H)^{l-\mu}}{l ! h^{-\mu} 2^{\mu+l} \Gamma(\mu) H^{\mu+l}[\Gamma(\mu+l)]^{-1}} \\
& -\sum_{l=0}^{\mu-1} \sum_{i=0}^{\mu-l-1} \frac{h^{\mu} \Gamma(\mu+l) \mu^{i} z^{i}}{l ! i ! 2^{\mu+l-i} \bar{\gamma}^{i} \Gamma(\mu) H^{\mu+l}} \\
& \times\left\{\frac{(-1)^{l}(h-H)^{l+i-\mu}}{\exp \left(\frac{2(h-H) \mu z}{\bar{\gamma}}\right)}+\frac{(-1)^{\mu}(h+H)^{l+i-\mu}}{\exp \left(\frac{2(h+H) \mu z}{\bar{\gamma}}\right)}\right\} .
\end{aligned}
$$

By substituting the CDF of the $\eta-\mu$ distribution in (13) and the PDF of the $\kappa-\mu$ distribution in (4) into (10), the CDF of $\Gamma_{D}, F_{Z}(z)$ can be expressed as

$$
\begin{aligned}
F_{Z}(z) & = \\
& \int_{0}^{\infty}\left(\sum_{l=0}^{\mu_{R, D}-1} \frac{h_{R, D}^{\mu_{R, D}}(-1)^{l}\left(h_{R, D}-H_{R, D}\right)^{l-\mu_{R, D}}}{l ! 2^{\mu_{R, D}+l}\left(\Gamma\left(\mu_{R, D}+l\right)^{-1} \Gamma\left(\mu_{R, D}\right) H_{R, D}^{\mu_{R, D}+l}\right.}\right. \\
& +\frac{h_{R, D}^{\mu_{R, D}}(-1)^{\mu_{R, D}}\left(h_{R, D}+H_{R, D}\right)^{l-\mu_{R, D}}}{l ! 2^{\mu_{R, D}+l}\left(\Gamma\left(\mu_{R, D}+l\right)\right)^{-1} \Gamma\left(\mu_{R, D}\right) H_{R, D}^{\mu_{R, D}+l}} \\
& -\sum_{l=0}^{\mu_{R, D}-1} \sum_{i=0}^{\mu_{R, D}-l-1} \frac{h_{R, D}^{\mu_{R, D}} \Gamma\left(\mu_{R, D}+l\right) \mu_{R, D}^{i}(z(x+1))^{i}}{l ! i ! 2^{\mu_{R, D}+l-i} \bar{\gamma}_{R, D}^{i} \Gamma\left(\mu_{R, D}\right) H_{R, D}^{\mu_{R, D}+l}} \\
\times & \left\{\frac{(-1)^{l}\left(h_{R, D}-H_{R, D}\right)^{l+i-\mu_{R, D}}}{\exp \left(\frac{2\left(h_{R, D}-H_{R, D}\right) \mu_{R, D} z(x+1)}{\bar{\gamma}_{R, D}}\right)}\right. \\
+ & \left.\left.\frac{(-1)^{\mu_{R, D}\left(h_{R, D}+H_{R, D}\right)^{l+i-\mu_{R, D}}}}{\exp \left(\frac{2\left(h_{R, D}+H_{R, D}\right) \mu_{R, D}(z(x+1))}{\bar{\gamma}_{R, D}}\right)}\right\}\right) \\
& \times \frac{A x \frac{\left(\mu_{S, D}-1\right)}{2} I_{\mu_{S, D}-1}\left(2 \mu_{S, D} \sqrt{\frac{\kappa_{S, D}\left(\kappa_{S, D}+1\right) x}{\gamma_{S, D}}}\right)}{\exp \left(\mu_{S, D} \kappa_{S, D}+\frac{\mu_{S, D}\left(\kappa_{S, D}+1\right) x}{\bar{\gamma}_{S, D}}\right)} \mathrm{d} x
\end{aligned}
$$

where

$$
A=\frac{\mu_{S, D}\left(1+\kappa_{S, D}\right)^{\frac{\mu_{S, D}+1}{2}}}{\kappa_{S, D}^{\frac{\mu_{S, D}-1}{2}}} \bar{\gamma}_{S, D}^{\frac{\mu_{S, D}+1}{2}} .
$$

By also applying [69, eq. (1.111)] and after long but basic algebraic manipulations, equation (14) can be expressed according to (16), at the top of the next page, where

$$
B=\frac{h_{R, D}^{\mu_{R, D}} \mu_{S, D}\left(1+\kappa_{S, D}\right)^{\frac{\mu_{S, D}+1}{2}}}{\Gamma\left(\mu_{R, D}\right) \kappa_{S, D}^{\frac{\mu_{S, D}-1}{S}} \exp \left(\kappa_{S, D} \mu_{S, D}\right) \bar{\gamma}_{S, D}^{\frac{\mu_{S, D}+1}{2}}} .
$$

Importantly, the involved integrals in (16) can be expressed in a closed form with the aid of [69, eq. (6.621.1)]. Therefore, by performing the necessary variable transformations and carrying out long but basic algebraic operations, the CDF of $\Gamma_{D}$ can be expressed according to (18), at the top of the next page. It is noted here that the derived expression can be readily substituted in (7) for deriving a novel closedform expression for the OP of the considered FD DF relay system over generalized asymmetric fading conditions. This expression is long but relatively convenient to handle both analytically and numerically as it involves elementary and special functions that are built-in in most popular scientific software packages such as MATLAB, MAPLE and MATHEMATICA. In addition, given that the derived closed-form expression is exact, it can form the basis for the derivation of simpler asymptotic and/or approximative expressions that can provide additional insights on the performance of the considered system and the effects of the involved parameters.

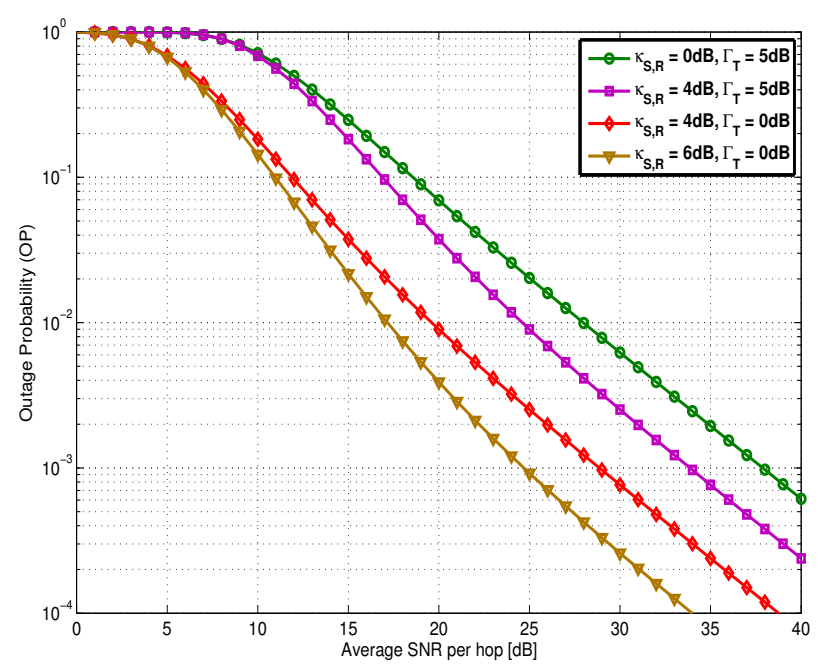

Fig. 2. Outage probability of full-duplex relaying vs. average SNR over asymmetric $\kappa-\mu$ and $\eta-\mu$ and fading channels for $\mu_{S, R}=\mu_{R, D}=$ $\mu_{S, D}=1, \eta_{R, D}=1, \kappa_{S, D}=2 \mathrm{~dB}, \bar{\gamma}_{R, R}=-5 \mathrm{~dB}, \bar{\gamma}_{S, D}=-10 \mathrm{~dB}$ and different values of $\kappa_{S, R}$ and $\Gamma_{T}$.

\section{Numerical Results AND Discussion}

In this section, we employ the derived analytic results for evaluating the performance of the considered DF-FDR system over asymmetric generalized fading conditions for different fading and interference scenarios. Furthermore, it is assumed that the transmit power of the system is allocated equally to the source and relay nodes i.e. $\bar{\gamma}=\bar{\gamma}_{S, R}=\bar{\gamma}_{R, D}$.

Fig.2 illustrates the OP behavior of the FD system where the $S \rightarrow R$ and $S \rightarrow D$ links are subject to $\kappa-\mu$ fading model with fading parameters $\mu_{S, R}=1$ and $\kappa_{S, R}=\{0,4,6\} \mathrm{dB}$, $\mu_{S, D}=1$ and $\kappa_{S, D}=2 \mathrm{~dB}$. Likewise, the $R \rightarrow D$ link is subject to $\eta-\mu$ fading conditions with $\mu_{R, D}=1$ and $\eta_{R, D}=1$. The average SNR values of the relay and the direct interference are set to be $\bar{\gamma}_{R, R}=-5 \mathrm{~dB}$ and $\bar{\gamma}_{S, D}=-10 \mathrm{~dB}$, respectively, for two SINR thresholds, namely $\Gamma_{T}=0 \mathrm{~dB}$ and $\Gamma_{T}=5 \mathrm{~dB}$. It is observed that as the $\kappa_{S, R}$ parameter increases, the corresponding outage events reduce, for a fixed $\Gamma_{T}$. Furthermore, the OP degrades as the target $\Gamma_{T}$ increases. 


$$
\begin{aligned}
& F_{Z}(z)=\sum_{l=0}^{\mu_{R, D}^{-1}} \frac{B\left((-1)^{l}\left(h_{R, D}-H_{R, D}\right)^{l-\mu_{R, D}}+(-1)^{\mu_{R, D}}\left(h_{R, D}+H_{R, D}\right)^{l-\mu_{R, D}}\right)}{\left(\Gamma\left(\mu_{R, D}+l\right)\right)^{-1} l ! 2^{\mu_{R, D}+l} H_{R, D}^{\mu_{R, D}+l}} \int_{0}^{\infty} \frac{I_{\mu_{S, D}-1}\left(2 \mu_{S, D} \sqrt{\frac{\kappa_{S, D}\left(\kappa_{S, D}+1\right) x}{\bar{\gamma}_{S, D}}}\right)}{x^{\frac{1-\mu_{S, D}}{2}} \exp \left(\frac{\mu_{S, D}\left(1+\kappa_{S, D}\right) x}{\bar{\gamma}_{S, D}}\right)} \mathrm{d} x \\
& -\sum_{l=0}^{\mu_{R, D}-1} \sum_{i=0}^{\mu_{R, D}-l-1} \sum_{j=0}^{i}\left(\begin{array}{l}
i \\
j
\end{array}\right) \frac{B(-1)^{l} z^{i} \Gamma\left(\mu_{\mu_{R, D}}+l\right) \mu_{R, D}^{i}\left(h_{R, D}-H_{R, D}\right)^{l+i-\mu_{R, D}}}{l ! i ! 2^{\mu_{R, D}+l-i} \bar{\gamma}_{R, D}^{i} H_{R, D}^{\mu_{R, D}+l} \exp \left(\frac{2\left(h_{R, D}-H_{R, D}\right) z \mu_{R, D}}{\bar{\gamma}_{R, D}}\right)} \\
& \times \int_{0}^{\infty} x^{\frac{\mu_{S, D}-1}{2}+j} I_{\mu_{S, D}-1}\left(2 \mu_{S, D} \sqrt{\frac{\kappa_{S, D}\left(\kappa_{S, D}+1\right) x}{\bar{\gamma}_{S, D}}}\right) \exp \left(-x\left(\frac{\mu_{S, D}\left(1+\kappa_{S, D}\right)}{\bar{\gamma}_{S, D}}+\frac{2\left(h_{R, D}-H_{R, D}\right) z \mu_{R, D}}{\bar{\gamma}_{R, D}}\right)\right) \mathrm{d} x \\
& -\sum_{l=0}^{\mu_{R, D}-1} \sum_{i=0}^{\mu_{R, D}-l-1} \sum_{j=0}^{i}\left(\begin{array}{l}
i \\
j
\end{array}\right) \frac{B(-1)^{\mu_{R, D}} z^{i} \Gamma\left(\mu_{\mu_{R, D}}+l\right) \mu_{R, D}^{i}\left(h_{R, D}+H_{R, D}\right)^{l+i-\mu_{R, D}}}{l ! i ! 2^{\mu_{R, D}+l-i} \bar{\gamma}_{R, D}^{i} H_{R, D}^{\mu_{R, D}+l} \exp \left(\frac{2\left(h_{R, D}+H_{R, D}\right) z \mu_{R, D}}{\bar{\gamma}_{R, D}}\right)} \\
& \times \int_{0}^{\infty} x^{\frac{\mu_{S, D}-1}{2}+j} I_{\mu_{S, D}-1}\left(2 \mu_{S, D} \sqrt{\frac{\kappa_{S, D}\left(\kappa_{S, D}+1\right) x}{\bar{\gamma}_{S, D}}}\right) \exp \left(-x\left(\frac{\mu_{S, D}\left(1+\kappa_{S, D}\right)}{\bar{\gamma}_{S, D}}+\frac{2\left(h_{R, D}+H_{R, D}\right) z \mu_{R, D}}{\bar{\gamma}_{R, D}}\right)\right) \mathrm{d} x
\end{aligned}
$$

$$
\begin{aligned}
F_{Z}(z) & =\sum_{l=0}^{\mu_{R, D}-1} \frac{\left((-1)^{l}\left(h_{R, D}-H_{R, D}\right)^{l-\mu_{R, D}}+(-1)^{\mu_{R, D}}\left(h_{R, D}+H_{R, D}\right)^{l-\mu_{R, D}}\right)}{h_{R, D}^{-\mu_{R, D}}\left(\Gamma\left(\mu_{R, D}+l\right)\right)^{-1} l ! 2^{\mu_{R, D}+l} \Gamma\left(\mu_{R, D}\right) H_{R, D}^{\mu_{R, D}+l}} \\
& -\sum_{l=0}^{\mu_{R, D}-1} \sum_{i=0}^{\mu_{R, D}-l-1} \sum_{j=0}^{i}\left(\begin{array}{l}
i \\
j
\end{array}\right) \frac{z^{i} \Gamma\left(\mu_{R, D}+l\right) \mu_{R, D}^{i} \mu_{S, D}^{\mu_{S, D}} \bar{\gamma}_{R, D}^{\mu_{S, D}+j-i} \bar{\gamma}_{S, D}^{j} \Gamma\left(\mu_{S, D}+j\right)}{h_{R, D}^{-\mu_{R, D}} \exp \left(\kappa_{S, D} \mu_{S, D}\right) \Gamma\left(\mu_{S, D}\right) l ! i ! 2^{\mu_{R, D}+l-i} \Gamma\left(\mu_{R, D}\right) H_{R, D}^{\mu_{R, D}+l}} \\
\times & \left\{\frac{\left((-1)^{l}\left(h_{R, D}-H_{R, D}\right)^{l+i-\mu_{R, D}}\left(1+\kappa_{S, D}\right)^{\mu_{S, D}}{ }_{1} F_{1}\left(\mu_{S, D}+j, \mu_{S, D}, \frac{\mu_{S, D}^{2} \bar{\gamma}_{R, D} \kappa_{S, D}\left(1+\kappa_{S, D}\right)}{\mu_{S, D} \bar{\gamma}_{R, D}\left(1+\kappa_{S, D}\right)+2 \bar{\gamma}_{S, D}\left(h_{R, D}-H_{R, D}\right) z \mu_{R, D}}\right)\right)}{\exp \left(\frac{2\left(h_{R, D}-H_{R, D}\right) z \mu_{R, D}}{\bar{\gamma}_{R, D}}\right)\left(\mu_{S, D} \bar{\gamma}_{R, D}\left(1+\kappa_{S, D}\right)+2\left(h_{R, D}-H_{R, D}\right) \bar{\gamma}_{S, D} z \mu_{R, D}\right)^{\mu_{S, D}+j}}\right. \\
+ & \frac{\left((-1)^{\mu_{R, D}}\left(h_{R, D}+H_{R, D}\right)^{l+i-\mu_{R, D}}\left(1+\kappa_{S, D}\right)_{1} F_{1}\left(\mu_{S, D}+j, \mu_{S, D}, \frac{\mu_{S, D}^{2} \bar{\gamma}_{R, D} \kappa_{S, D}\left(1+\kappa_{S, D}\right)}{\mu_{S, D} \bar{\gamma}_{R, D}\left(1+\kappa_{S, D}\right)+2 \bar{\gamma}_{S, D}\left(h_{R, D}+H_{R, D}\right) z \mu_{R, D}}\right)\right)}{\exp \left(\frac{2\left(h_{R, D}+H_{R, D}\right) z \mu_{R, D}}{\bar{\gamma}_{R, D}}\right)\left(\mu_{S, D} \bar{\gamma}_{R, D}\left(1+\kappa_{S, D}\right)+2\left(h_{R, D}+H_{R, D}\right) \bar{\gamma}_{S, D} z \mu_{R, D}\right)^{\mu_{S, D}+j}}
\end{aligned}
$$

This is because, the system remains in complete outage until the target SINR is achieved for successful transmission.

Fig.3 depicts the outage probability of the FD system as a function of the average SNR with target SINRs of $\Gamma_{T}=$ $0 \mathrm{~dB}$ and $\Gamma_{T}=5 \mathrm{~dB}$ for $\kappa_{S, R}=4 \mathrm{~dB}, \mu_{S, D}=1, \kappa_{S, D}=2 \mathrm{~dB}$, $\eta_{R, D}=1, \bar{\gamma}_{S, D}=-10 \mathrm{~dB}$ with different values of $\mu_{S, R}, \mu_{R, D}$ and relay self-interference $\bar{\gamma}_{R, R}$. It is observed that the outage performance improves when the required target $\Gamma_{T}$ decreases. Furthermore, it is noticed that at the lower portion of the highSNR regime, the RSI degrades the outage probability of the system even for low fading severity. However, this changes dramatically in the case of the higher portion of the high SNR regime. For example, the OP for $\Gamma_{T}=5 \mathrm{~dB}$ with fading parameters of $\mu_{S, R}=\mu_{R, D}=3$ with $\bar{\gamma}_{R, R}=4 \mathrm{~dB}$ performs better than in the case of $\mu_{S, R}=\mu_{R, D}=2$ and $\bar{\gamma}_{R, R}=0 \mathrm{~dB}$ beyond $25 \mathrm{~dB}$. This is also the case for average SNRs beyond $20 \mathrm{~dB}$ and target SINR at 0dB.

Finally, Fig. 4 demonstrates the effect of the direct $S \rightarrow D$ interference on the behavior and performance of the outage probability for fading parameters of $\mu_{S, R}=2, \kappa_{S, R}=4 \mathrm{~dB}$,
$\mu_{S, D}=1, \kappa_{S, D}=2 \mathrm{~dB}, \mu_{R, D}=1, \eta_{R, D}=1, \bar{\gamma}_{R, R}=-10 \mathrm{~dB}$ and different values of $\bar{\gamma}_{S, D}$ with target SINRs of $\Gamma_{T}=0 \mathrm{~dB}$ and $5 \mathrm{~dB}$, respectively. It is observed that the outage performance of the system is, as expected, significantly affected by the interference from the S-D link. This is because as the direct interference increases, the equivalent SINR at the destination is reduced, which ultimately degrades the corresponding outage probability.

\section{CONCLuSion}

This work analyzed the outage probability of a dualhop full-duplex regenerative system with relay and direct interference over asymmetric generalized multipath fading channels. Novel analytic expressions were derived for the outage probability for the case that the source-to-relay and source-to-destination paths experience $\kappa-\mu$ fading conditions whereas the relay-to-destination path experiences $\eta-\mu$ fading conditions. It was demonstrated that the performance of the considered cooperative system is affected significantly by the fading conditions in each communication path as well as by 


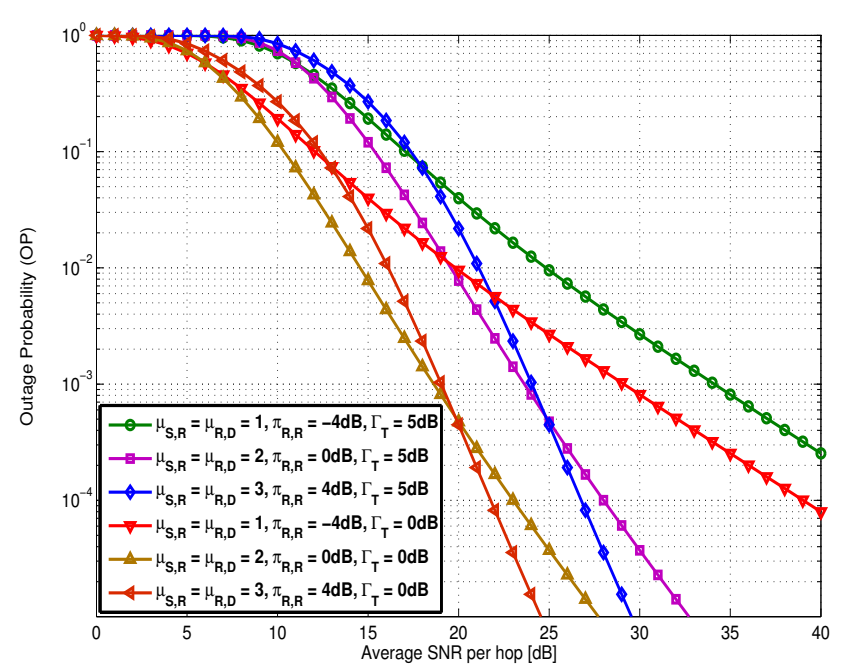

Fig. 3. Outage probability of full-duplex relaying vs. average SNR $(\bar{\gamma})$ over asymmetric $\kappa-\mu$ and $\eta-\mu$ fading channels for $\kappa_{S, R}=4 \mathrm{~dB}, \kappa_{S, D}=$ $2 \mathrm{~dB}, \mu_{S, D}=1, \eta_{R, D}=1, \bar{\gamma}_{S, D}=-10 \mathrm{~dB}$ for different values of $\mu_{S, R}, \mu_{R, D}, \bar{\gamma}_{R, R}=\pi_{R, R}$, and $\Gamma_{T}$.

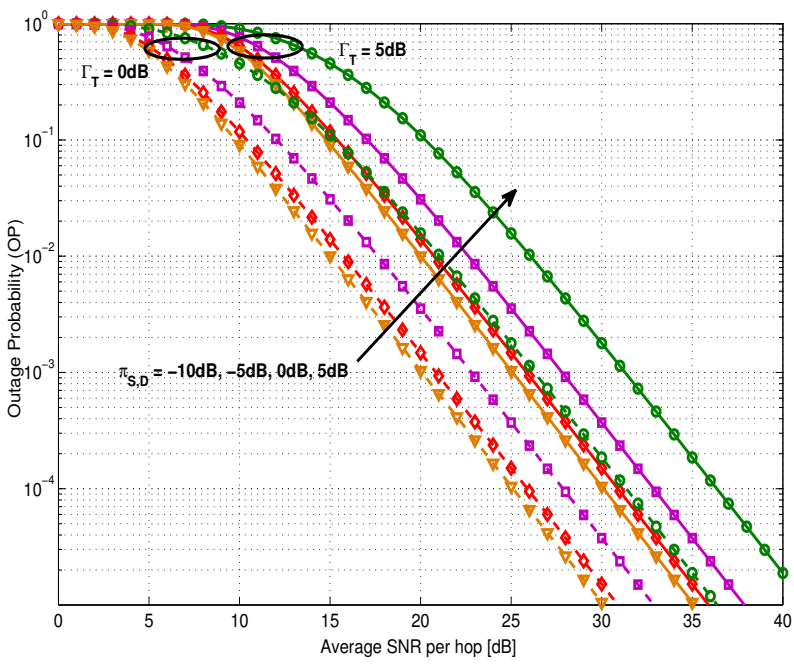

Fig. 4. Outage probability of full-duplex relaying vs. average SNR $(\bar{\gamma})$ over asymmetric $\kappa-\mu$ and $\eta-\mu$ fading channels for $\mu_{S, R}=2, \mu_{R, D}=\mu_{S, D}=$ $1, \eta_{R, D}=1, \kappa_{S, R}=4 \mathrm{~dB}, \kappa_{S, D}=2 \mathrm{~dB}, \bar{\gamma}_{R, R}=-10 \mathrm{~dB}$ and different values of $\bar{\gamma}_{S, D}=\pi_{S, D}$ and $\Gamma_{T}$.

the relay and direct interference. In addition, it is shown that in the high-SNR regime, the outage probability at low fading severity and at high relay self interference outperforms the respective performance for the case of high fading severity, but with low relay self-interference levels.

\section{ACKNOWLEDGEMENTS}

This work was supported by the Finnish Funding Agency for Technology and Innovation (Tekes) under the project entitled "Energy-Efficient Wireless Networks and Connectivity of Devices-Systems (EWINE-S)", by the Academy of Finland under the projects No. 251138 "Digitally-Enhanced RF for Cognitive Radio Devices and No. 284694 "Fundamentals of Ultra Dense 5G Networks with Application to Machine Type Communication" and by the National Nature Science Foundation of China Project "Grant No. 61471058".

\section{REFERENCES}

[1] Y. Yang, H. Hu, J. Xu, and G. Mao,"Relay technologies for WiMax and LTE-advanced mobile systems," IEEE Commun. Mag., vol. 47, no. 10, pp. 100-105, Oct. 2009.

[2] P. L. Yeoh, M. Elkashlan, Z. Chen, and I. B. Collings, "SER of multiple amplify-and-forward relays with selection diversity," IEEE Trans. Commun., vol. 59, no. 8, pp. 2078-2083, Aug. 2011.

[3] T. Q. Duong, D. B. da Costa, M. Elkashlan, and V. N. Q. Bao, "Cognitive amplify-and-forward relay networks over Nakagami- $m$ fading," IEEE Trans. Veh. Technol., vol. 61, no. 5, pp. 2368-2374, June 2012.

[4] R. Cao, and L. Yang, "Decomposed LT codes for cooperative relay communications," IEEE J. Sel. Areas Commun., vol. 30, no. 2, pp. 407-414, Feb. 2012.

[5] C. Zhong, H. A. Suraweera, A. Huang, Z. Zhang and C. Yuen, "Outage probability of dual-hop multiple antenna AF relaying systems with interference," IEEE Trans. Commun., vol. 61, no. 1, pp. 108-119, Jan. 2013.

[6] P. L. Yeoh, M. Elkashlan, T. Q. Duong, N. Yang, and D. B. da Costa, "Transmit antenna selection for interference management in cognitive relay networks," IEEE Trans. Veh. Technol., vol. 63, no. 7, p. 3250-3262, Sep. 2014.

[7] T. T. Duy, T. Q. Duong, D. B. da Costa, V. N. Q. Bao, and M. Elkashlan, "Proactive relay selection with joint impact of hardware impairment and co-channel interference," IEEE Trans. Commun., vol. 63, no. 5, pp. 1594-1606, May 2015.

[8] A. Nosratinia, T. E. Hunter, and A. Hedayat, "Cooperative communication in wireless networks," IEEE Commun. Mag., vol. 42, no. 10, pp. 74-80, Oct. 2004.

[9] H. A. Suraweera, P. J. Smith and J. Armstrong, "Outage probability of cooperative relay networks in Nakagami- $m$ fading channels," IEEE Commun. Lett., vol. 10, no. 12, pp. 834-836, Dec. 2006.

[10] K. Ho-Van, P. C. Sofotasios, and S. Freear, "Underlay cooperative cognitive networks with imperfect Nakagami $-m$ fading channel information and strict transmit power constraint: interference statistics and outage probability analysis," IEEE/KICS J. Commun. Networks, vol. 16, no. 1, pp. 10-17, Feb. 2014.

[11] Z. Ding, M. Peng and H. V. Poor, "Cooperative Non-Orthogonal Multiple Access in 5G Systems," IEEE Commun. Lett., to appear in 2015.

[12] K. Ho-Van, P. C. Sofotasios, G. C. Alexandropoulos, and S. Freear, "Bit error rate of underlay decode-and-forward cognitive networks with best relay selection," IEEE/KICS J. Commun. Networks, vol. 17, no. 2, pp. 162-171, Apr. 2015.

[13] T. T. Duy, T. Q. Duong, D. B. da Costa, V. N. Q. Bao, and M. Elkashlan, "Proactive relay selection with joint impact of hardware impairment and co-channel interference," IEEE Trans. Commun., vol. 63, no. 5, pp. 1594-1606, May 2015.

[14] M. K. Fikadu, P. C. Sofotasios, Q. Cui, M. Valkama, and G. K. Karagiannidis, "Exact error analysis and energy-efficiency optimization of regenerative relay systems," IEEE Trans. Veh. Technol., Accepted for Publication.

[15] K. Ho-Van, and P. C. Sofotasios, "Exact BER analysis of underlay decode-and-forward multi-hop cognitive networks with estimation errors," IET Commun., vol. 7, no. 18, pp. 2122-2132, Dec. 2013.

[16] G. C. Alexandropoulos, P. C. Sofotasios, K. Ho-Van, and S. Freear, "Symbol error probability of DF relay selection over arbitrary Nakagami $-m$ fading channels," HINDAWI Journal of Engineering, vol. 2013, Article ID 325045, 2013.

[17] M. K. Fikadu, P. C. Sofotasios, M. Valkama, Q. Cui, S. Muhaidat, and G. $\mathrm{K}$. Karagiannidis, "Outage probability analysis of full-duplex regenerative relaying over generalized asymmetric fading channels," in IEEE Globecom '15, San Diego, CA, USA, Dec. 2015. 
[18] G. C. Alexandropoulos, A. Papadogiannis, and P. C. Sofotasios, "A comparative study of relaying schemes with decode-and-forward over Nakagami $-m$ fading channels," Journal of Computer Networks and Communications, vol. 2011, Article ID 560528, Dec. 2011.

[19] M. K. Fikadu, P. C. Sofotasios, M. Valkama, S. Muhaidat, Q. Cui, and G. K. Karagiannidis, "Outage probability analysis of dual-hop fullduplex decode-and-forward relaying over generalized multipath fading conditions," in IEEE WiMob '15, Abu Dhabi, UAE, Oct. 2015.

[20] K. Ho-Van, and P. C. Sofotasios, "Bit error rate of underlay multi-hop cognitive networks in the presence of multipath fading," in IEEE ICUFN '13, Da Nang, Vietnam, July 2013.

[21] M. K. Fikadu, P. C. Sofotasios, M. Valkama, Q. Cui, S. Muhaidat, and G. K. Karagiannidis, "Analytic symbol error rate evaluation of $M$-PSK based regenerative cooperative networks over generalized fading channels," in IEEE WiMob '15, Abu Dhabi, UAE, Oct. 2015.

[22] K. Ho-Van, P. C. Sofotasios, V. Que Son, L. Thanh Tra, and P. Hong Lien, "Analysis of cognitive cooperative networks with best relay selection and diversity reception," in IEEE ATC '15, HoChiMinh City, Vietnam, Oct. 2015.

[23] L. Mohjazi, D. Dawoud, P. C. Sofotasios, S. Muhaidat, M. Dianati, M. Valkama, and G. K. Karagiannidis, "Unified analysis of cooperative spectrum sensing over generalized multipath fading channels," in IEEE PIMRC '15, Hong Kong, Aug/Sep. 2015.

[24] M. K. Fikadu, P. C. Sofotasios, M. Valkama, Q. Cui, and G. K. Karagiannidis, "Energy-efficiency analysis of regenerative cooperative systems under spatial correlation," in IEEE PIMRC '15, Hong Kong, Aug/Sep. 2015.

[25] K. Ho-Van, P. C. Sofotasios, S. V. Que, T. D. Anh, T. P. Quang, and L. P. Hong, "Analytic performance evaluation of underlay relay cognitive networks with channel estimation errors," in IEEE ATC '13, HoChiMinh City, Vietnam, Oct. 2013.

[26] M. K. Fikadu, P. C. Sofotasios, M. Valkama, and Q. Cui, "Analytic performance evaluation of $M$-QAM based decode-and-forward relay networks over enriched multipath fading channels," in IEEE WiMob '14, Larnaca, Cyprus, Oct. 2014.

[27] K. Ho-Van, and P. C. Sofotasios, "Outage behaviour of cooperative underlay cognitive networks with inaccurate channel estimation," in IEEE ICUFN '13, Da Nang, Vietnam, July 2013.

[28] Z. Mo, W. Su, S. Batalama, and J. D. Matyjas, "Cooperative communication protocol designs based on optimum power and time allocation," IEEE Trans. Wireless Commun., vol. 13, no. 8, pp. 4283-4296, Aug. 2014.

[29] M. R. Bhatnagar, "Decode-and-forward-based differential modulation for cooperative communication system with unitary and non unitary constellations," IEEE Trans. Veh. Techn., vol. 61, no. 1, pp. $152-165$, Jan. 2012.

[30] A. K. Sadek, W. Su, and K. J. R. Liu, "Multi-node cooperative communications in wireless networks," IEEE Trans. Signal Process., vol. 55, no. 1, pp. 341-355, Jan. 2007.

[31] Z. Ding, M. Peng and H. V. Poor, "Cooperative Non-Orthogonal Multiple Access in 5G Systems," IEEE Commun. Lett., to appear in 2015

[32] G. Zhu, C. Zhong, H. A. Suraweera, Z. Zhang, C. Yuen and R. Yin, "Ergodic capacity comparison of different relay precoding schemes in dualhop AF systems with co-channel interference," IEEE Trans. Commun., vol. 62, no. 7, pp. 2314-2328, July 2014.

[33] T. Riihonen, S. Werner, and R. Wichman, "Comparison of full-duplex and half-duplex modes with a fixed amplify-and-forward relay," in Proc. IEEE Wireless Commun. Netw. Conf., Apr. 2009, pp. 1-5.

[34] L. J. Rodrguez, N. H. Tran, and T. Le-Ngoc, "Performance of fullduplex AF relaying in the presence of residual self-interference," IEEE $J$. Sel. Areas Commun., vol. 32, no. 9, pp. 1752-1764, Sep. 2014.

[35] C. Zhong, H. A. Suraweera, G. Zheng, I. Krikidis and Z. Zhang, "Wireless information and power transfer with full duplex relaying," IEEE Trans. Commun., vol. 62, no. 10, pp. 3447-3461, Oct. 2014.

[36] Z. Zhang, X. Chai, K. Long, A. V. Vasilakos, and L. Hanzo, " Full Duplex techniques for 5G networks: Self-interference cancellation, protocol design, and relay selection," IEEE Commun. Mag., vol. 53, no. 5, pp. 128-137, May 2015.

[37] M. Duarte, C. Dick, and A. Sabharwal," Experiment-driven characterization of full-duplex wireless systems," IEEE Trans. Wireless Commun., vol. 11 , no. 12, 4296-4307, Dec. 2012.

[38] T. Riihonen, S. Werner, and R. Wichman," Optimized gain control for single-frequency relaying with loop interference," IEEE Trans. Wireless Commun., vol. 8, pp. 2801-2806, June 2009.

[39] T. K. Baranwal, and D. S. Michalopoulos, "Outage analysis of multihop full duplex relaying," IEEE Commun. Lett, vol. 17, no. 1, pp. 63-66, Jan. 2013.

[40] B. P. Day, A. R. Margetts, D. W. Bliss, and P. Schniter, "Full-duplex MIMO relaying: Achievable rates under limited dynamic range," IEEE $J$. Sel. Areas Commun., vol. 30, no. 8, pp. 1541-1553, Sep. 2012.

[41] A. Altieri, L. R. Vega, P. Piantanida, and C. G. Galarza, "On the outage probability of the full-duplex interference-limited relay channel," IEEE $J$. Sel. Areas Commun., vol. 32, no. 9, pp. 1765-1777, Sep. 2014.

[42] S. Ki Yoo, S. L. Cotton, P. C. Sofotasios, M. Matthaiou, M. Valkama, and G. K. Karagiannidis, "The $\kappa-\mu$ / Inverse gamma fading model," in IEEE PIMRC '15, Hong Kong, Aug/Sep. 2015.

[43] S. Ki Yoo, P. C. Sofotasios, S. L. Cotton, M. Matthaiou, M. Valkama, and G. K. Karagiannidis, "The $\eta-\mu /$ Inverse gamma composite fading model," in IEEE PIMRC '15, Hong Kong, Aug/Sep. 2015.

[44] P. C. Sofotasios, T. A. Tsiftsis, K. Ho-Van, S. Freear, L. R. Wilhelmsson, and M. Valkama, "The $\kappa-\mu /$ inverse-Gaussian composite statistical distribution in RF and FSO wireless channels," in IEEE VTC '13 - Fall, Las Vegas, USA, Sep. 2013.

[45] P. C. Sofotasios, T. A. Tsiftsis, M. Ghogho, L. R. Wilhelmsson, and M.Valkama, "The $\eta-\mu /$ inverse Gaussian distribution: A novel physical multipath/shadowing fading model," in IEEE ICC' 13, Budapest, Hungary, June 2013.

[46] P. C. Sofotasios, and S. Freear, "The $\alpha-\kappa-\mu /$ gamma composite distribution: A generalized non-linear multipath/shadowing fading model," in IEEE INDICON '11, Hyderabad, India, Dec. 2011.

[47] P. C. Sofotasios, and S. Freear, "The $\alpha-\kappa-\mu$ extreme distribution: characterizing non linear severe fading conditions," in ATNAC '11, Melbourne, Australia, Nov. 2011.

[48] P. C. Sofotasios, and S. Freear, "The $\eta-\mu /$ gamma and the $\lambda-\mu /$ gamma multipath/shadowing distributions," in ATNAC '11, Melbourne, Australia, Nov. 2011.

[49] P. C. Sofotasios, and S. Freear, "On the $\kappa-\mu /$ gamma composite distribution: A generalized multipath/shadowing fading model," in IEEE IMOC '11, Natal, Brazil, Oct. 2011.

[50] S. Harput, P. C. Sofotasios, and S. Freear, "A novel composite statistical model for ultrasound applications," in IEEE IUS '11, Orlando, FL, USA, Oct. 2011.

[51] P. C. Sofotasios, and S. Freear, "The $\kappa-\mu /$ gamma extreme composite distribution: A physical composite fading model," in IEEE WCNC '11, Cancun, Mexico, Mar. 2011.

[52] P. C. Sofotasios, and S. Freear, "The $\kappa-\mu /$ gamma composite fading model,' in IEEE ICWITS '10, Honolulu, HI, USA, Aug/Sep. 2010.

[53] P. C. Sofotasios, and S. Freear, "The $\eta-\mu /$ gamma composite fading model," in IEEE ICWITS '10, Honolulu, HI, USA, Aug/Sep. 2010.

[54] P. C. Sofotasios, S. Muhaidat, G. K. Karagiannidis, and B. S. Sharif, "Solutions to integrals involving the Marcum $Q$-function and applications," IEEE Signal Process. Lett., vol. 22, no. 10, pp. 1752-1756, Oct. 2015.

[55] P. C. Sofotasios, T. A. Tsiftsis, Yu. A. Brychkov, S. Freear, M. Valkama, and G. K. Karagiannidis, "Analytic expressions and bounds for special functions and applications in communication theory," IEEE Trans. Inf. Theory, vol. 60, no. 12, pp. 7798-7823, Dec. 2014.

[56] P. C. Sofotasios, M. Valkama, Yu. A. Brychkov, T. A. Tsiftsis, S. Freear, and G. K. Karagiannidis, "Analytic solutions to a Marcum $Q$-functionbased integral and application in energy detection," in CROWNCOM 14, Oulu, Finland, June 2014.

[57] P. C. Sofotasios, K. Ho-Van, T. D. Anh, and H. D. Quoc, "Analytic results for efficient computation of the Nuttall $-Q$ and incomplete Toronto functions," in IEEE ATC '13, HoChiMinh City, Vietnam, Oct. 2013.

[58] P. C. Sofotasios, and S. Freear, "New analytic expressions for the Rice Function and the Incomplete Lipschitz-Hankel Integrals," in IEEE INDICON '11, Hyderabad, India, Dec. 2011.

[59] P. C. Sofotasios, and S. Freear, "Upper and lower bounds for the Rice Ie-function," in ATNAC '11, Melbourne, Australia, Nov. 2011.

[60] P. C. Sofotasios, and S. Freear, "Novel results for the incomplete Toronto function and incomplete Lipschitz-Hankel integrals," in IEEE IMOC '11, Natal, Brazil, Oct. 2011.

[61] P. C. Sofotasios, and S. Freear, "Simple and accurate approximations for the two dimensional Gaussian $Q$-function," in IEEE VTC-Spring '11, Budapest, Hungary, May 2011.

[62] P. C. Sofotasios, and S. Freear, "Novel expressions for the Marcum and one dimensional $Q$-functions," in 7th ISWCS '10, York, UK, Sep. 2010.

[63] P. C. Sofotasios, and S. Freear, "Novel expressions for the one and two dimensional Gaussian $Q$-functions," in IEEE ICWITS '10, Honolulu, HI, USA, Aug/Sep. 2010. 
[64] P. C. Sofotasios, and S. Freear, "A novel representation for the Nuttall $Q$-function," in IEEE ICWITS '10, Honolulu, HI, USA, Aug/Sep. 2010.

[65] H. A. Suraweera, G. K. Karagiannidis, and P. J. Smith, "Performance analysis of the dual-hop asymmetric fading channel," IEEE Trans. Wireless Commun., vol. 8, no. 6, pp. 2783-2788, June 2009.

[66] P. Jayasinghe, L. K. S. Jayasinghe, M. Juntti, and M. Latva-Aho, "Performance analysis of optimal beamforming in fixed-gain AF MIMO relaying over asymmetric fading channels," IEEE Trans. on Commun., vol. 62, no. 4, pp. 1201-1217, Apr. 2014.

[67] K. P. Peppas, G. C. Alexandropoulos, and P. T. Mathiopoulos, "Performance analysis of dual-hop AF relaying systems over mixed $\eta-\mu$ fading channels," IEEE Trans. Veh. Technol., vol. 62, no. 7, pp. 3149-3163, Sep.
2013.

[68] M. D. Yacoub, "The $\kappa-\mu$ distribution and the $\eta-\mu$ distribution," IEEE Ant. Propag. Mag., vol. 49, no. 1, pp. 68-81, Feb. 2007.

[69] I. S. Gradshteyn, and I. M. Ryzhik, Tables of Integrals, Series, and Products $-7^{\text {th }}$ edn. Academic Press, 2007.

[70] T. Kwon, S. Lim, S. Choi, and D. Hong, "Optimal duplex mode for DF relay in terms of the outage probability," IEEE Trans. Veh. Technol., vol. 59, no. 7, pp. 3628-3634, Sep. 2010.

[71] A. Papoulis, S.U. Pillai, Probability, Random Variables and Stochastic Processes, $-4^{\text {th }}$ edn. McGraw-Hill, 2002.

[72] A. H. Nuttall, "Some Integrals Involving the $Q_{M}$ Function," IEEE Inf. Theory, vol. 21, no. 1, pp. 95-96, 1975. 